\title{
Association of early HIV viremia with mortality after HIV- associated lymphoma
}

\author{
Satish Gopala, ${ }^{a}$, Monita R. Patela, ${ }^{a}$, Elizabeth L. Yanik ${ }^{a}$, Stephen R. Cole ${ }^{a}$, Chad J. \\ Achenbach $^{\mathrm{b}}$, Sonia Napravnik ${ }^{\mathrm{a}}$, Greer A. Burkholder ${ }^{\mathrm{c}}$, Erin G. Reid ${ }^{\mathrm{d}}$, Benigno Rodriguez ${ }^{\mathrm{e}}$, \\ Steven G. Deeks ${ }^{f}$, Kenneth H. Mayer ${ }^{g}$, Richard D. Moore ${ }^{h}$, Mari M. Kitahatai, Kristy L. \\ Richards $^{\mathrm{a}}$, and Joseph J. Eron ${ }^{\mathrm{a}}$
}

aUniversity of North Carolina at Chapel Hill, Chapel Hill, North Carolina bNorthwestern University, Chicago, Illinois 'University of Alabama at Birmingham, Birmingham, Alabama dUniversity of California at San Diego, San Diego, California ${ }^{\mathrm{e}}$ Case Western Reserve University, Cleveland, Ohio fUniversity of California at San Francisco, San Francisco, California 9Fenway Health, Boston, Massachusetts hJohns Hopkins University, Baltimore, Maryland iUniversity of Washington, Seattle, Washington

\section{Abstract}

Objective-To examine the association between early HIV viremia and mortality after HIVassociated lymphoma.

Design-Multicenter observational cohort study.

Setting-Center for AIDS Research Network of Integrated Clinical Systems cohort.

Subjects-HIV-infected patients with lymphoma diagnosed between 1996 and 2011, who were alive 6 months after lymphoma diagnosis and with $\geq 2$ HIV RNA values during the 6 months after lymphoma diagnosis.

Exposure-Cumulative HIV viremia during the 6 months after lymphoma diagnosis, expressed as viremia copy-6-months.

Main outcome measure-All-cause mortality between 6 months and 5 years after lymphoma diagnosis.

Results-Of 224 included patients, 183 (82\%) had non-Hodgkin lymphoma (NHL) and 41 (18\%) had Hodgkin lymphoma (HL). At lymphoma diagnosis, 105 (47\%) patients were on antiretroviral therapy (ART), median CD4 count was 148 cells/ $\mu$ IL (IQR 54- 322), and 33\% had suppressed HIV RNA ( $<400$ copies $/ \mathrm{mL}$ ). In adjusted analyses, mortality was associated with older age [adjusted hazard ratio (AHR) 1.37 per decade increase, 95\% CI 1.03-1.83], lymphoma occurrence on ART (AHR 1.63, 95\% CI 1.02- 2.63), lower CD4 count (AHR 0.75 per 100 cell/ $\mu \mathrm{L}$ increase, $95 \%$ CI $0.64-0.89$ ), and higher early cumulative viremia (AHR 1.35 per log 10copies $\times$ 6-months/mL, 95\% CI 1.11-1.65). The detrimental effect of early cumulative viremia was consistent across patient groups defined by ART status, CD4 count, and histology.

(C) 2013 Wolters Kluwer Health | Lippincott Williams \& Wilkins

Correspondence to Satish Gopal, MD, MPH; Program in Global Oncology, Lineberger Comprehensive Cancer Center, UNC ProjectMalawi, Tidziwe Center, Private Bag A-104, Lilongwe, Malawi. Tel: +265 1755 056; fax: +265 1755 954; gopal@ med.unc.edu.

*S.G. and M.R.P contributed equally to this study.

Conflicts of interest

Conflicts of interest and source of funding: No conflict of interest declared; National Institutes of Health, UNC Lineberger

Comprehensive Cancer Center 
Conclusions-Exposure to each additional 1 -unit $\log _{10}$ in HIV RNA throughout the 6 months after lymphoma diagnosis, was associated with a $35 \%$ increase in subsequent mortality. These results suggest that early and effective ART during chemotherapy may improve survival.

\section{Keywords}

AIDS; Burkitt lymphoma; diffuse large B-cell lymphoma; HIV; Hodgkin lymphoma; lymphoma; non-Hodgkin lymphoma

\section{Introduction}

In resource-rich settings, cancer is the leading cause of death among HIV-infected persons in the modern antiretroviral therapy (ART) era, and lymphoma the most frequent cancerrelated cause [1-4].

Effects of ART early after HIV-associated lymphoma diagnosis remain uncertain. Chemotherapy clinical trials conducted by the National Cancer Institute (NCI) and AIDS Malignancy Consortium (AMC) have employed various strategies with respect to ART without directly comparing approaches. Strategies have included concurrent ART during chemotherapy [5,6], ART suspension until chemotherapy completion [7,8], or ART left to physicians' discretion [9]. Potential negative effects of concurrent ART during chemotherapy include overlapping toxicities, medication interactions [10,11], antiapoptotic ART effects [10,12,13], and HIV resistance from ART interruption [10].

Similar concerns previously existed regarding ART during treatment for opportunistic infections (OIs), including toxicities and interactions, ART discontinuity and HIV resistance, and risk of the immune reconstitution inflammatory syndrome (IRIS). However, multiple randomized clinical trials have demonstrated that deferring ART by even several weeks worsens outcomes for patients with OIs, particularly when severely immunosuppressed, with cryptococcal and tuberculous meningitis being possible exceptions [14-19]. A similar detrimental effect of deferred ART is possible for HIV-associated lymphoma patients, who present with significant immunosuppression even in the ART era [5- 9,20,21]. Additionally, Epstein-Barr virus (EBV) is present in approximately $40 \%$ of tumors with marked variation by histologic subtype [10]. For EBV-associated lymphomas, early immune reconstitution may improve outcomes, analogous to EBV-associated posttransplant lymphoproliferative disease (PTLD), for which prompt reduction of immunosuppression may obviate need for chemoimmunotherapy [22].

Reflecting these controversies, consensus guidelines make no strong recommendation for or against concurrent ART during chemotherapy [22]. We studied a large United States cohort of HIV-infected lymphoma patients who survived at least six months after lymphoma diagnosis, to examine effects of early HIV viremia, as a marker of early and effective ART, on all-cause mortality.

\section{Methods}

\section{Patients}

The Center for AIDS Research (CFAR) Network of Integrated Clinical Systems (CNICS) cohort has been described elsewhere [23]. CNICS provides a clinical data repository from electronic medical record systems. CNICS includes over 25,000 HIV-infected adults older than 18 years, receiving care from January 1, 1995 to the present, at eight CFAR sites throughout the United States (Case Western Reserve University; University of Alabama at Birmingham; University of California, San Francisco; University of Washington; University 
of California, San Diego; Fenway Health, affiliated with Harvard University; University of North Carolina; Johns Hopkins University).

We examined individuals in CNICS with non-Hodgkin lymphoma (NHL) or Hodgkin lymphoma (HL) diagnosed between January 1, 1996 and December 31, 2011. If more than one lymphoma diagnosis or relapse was recorded for a single patient, we analyzed only the first occurrence. For inclusion, patients were additionally required to have at least two unique HIV RNA measurements taken at least one month apart, during the time interval between one month before and seven months after lymphoma diagnosis. To avoid excluding patients with HIV RNA values taken just before or after the six-month window of interest following lymphoma diagnosis, a one-month tail was included on either side. Patients were also required to be at risk for mortality beginning six months after lymphoma diagnosis (i.e. not deceased, lost to follow-up, or administratively censored prior to this time point). All inclusion criteria were intended to allow reliable assessment of the principal exposure of interest, cumulative HIV viremia during the six months after lymphoma diagnosis.

\section{Procedures}

Upon CNICS entry, standardized demographic and historical information, including prior diagnoses and antiretroviral treatment, are collected. Data including medications, laboratory values, and AIDS-defining and non-AIDS-defining conditions, are prospectively collected and verified by medical record review. A standardized cancer diagnosis verification procedure has been established [24]. Incident cancers are reviewed for confirmation and to collect information regarding type, histology, staging, and treatment. Patients must attend HIV clinics at network sites to be enrolled, although data may be available from electronic medical records prior to cohort entry. Mortality data are obtained from clinic sources and confirmed by the Social Security Death Index (SSDI). CD4 count and HIV RNA at lymphoma diagnosis were defined as values closest to diagnosis date beginning three months before until three months after. Nadir CD4 count was defined as the lowest CD4 count at any time on or before the date of CD4 at lymphoma diagnosis. Undetectable HIV RNA was assigned a value equal to half the detection limit of the assay. Suppressed HIV RNA was defined as less than 400 copies $/ \mathrm{mL}$. Hepatitis B co-infection was defined as any positive hepatitis B surface antigen or DNA result, and hepatitis C co-infection as any positive hepatitis $\mathrm{C}$ antibody or RNA result, before or until six months after lymphoma diagnosis. Lymphoma developing on ART was defined as receipt of any antiretroviral medication from six months to one month before lymphoma diagnosis. Patients with ART exposure ending more than six months prior to lymphoma diagnosis, or ART initiated within one month prior to lymphoma diagnosis, were classified as being off ART at lymphoma diagnosis.

\section{Statistical analysis}

We assessed cumulative HIV viremia using the viremia copy-years method [25,26]. Given the six-month window of interest, we adapted this to define our principal exposure as viremia copy-6-months. This is a measure of cumulative HIV viremia during the six months after lymphoma diagnosis, similar to smoking pack-years. The trapezoidal rule is used to approximate the integral representing the area under the longitudinal HIV RNA curve for each patient. HIV RNA burden for each time interval between two consecutive HIV RNA values is calculated by multiplying the mean of the two values by the time interval. The copy x 6-months/mL values for each segment of a patient's HIV RNA curve are then summed to calculate viremia copy-6-months. For instance, having a viremia copy-6-months value of $3 \log _{10}$ copies $x$ 6-months $/ \mathrm{mL}$ is equivalent to having an HIV RNA of 1000 copies/ $\mathrm{mL}$ for six months, or an HIV RNA of 2000 copies $/ \mathrm{mL}$ for two months followed by 500 copies/mL for four months. If the first HIV RNA measure occurred before lymphoma 
diagnosis, this was carried forward to the lymphoma diagnosis date. If the first HIV RNA measure occurred after lymphoma diagnosis, this was carried backward to the lymphoma diagnosis date. Similarly, if the last HIV RNA measure occurred prior to six months after lymphoma diagnosis, this was carried forward, and if the last HIV RNA measure occurred after, it was carried backward to the six-month time point. For all patients, area under the HIV RNA curve was calculated for the entire six-month window of interest. We additionally examined effects of cross-sectional HIV RNA measures at lymphoma diagnosis and six months after.

Differences in proportions, means, and medians for patients developing lymphoma on and off ART were assessed using chi-square or Fisher's exact tests, one-way analysis of variance (ANOVA), and Kruskal-Wallis tests, respectively. HIV RNA curves for patients on and off ART at lymphoma diagnosis were constructed by taking the median HIV RNA value across all patients at each monthly time point. HIV RNA values for individual patients at each monthly time point were assigned based on the value at which the individual's HIV RNA curve intersected each time point similar to the segmental viremia copy-years method described above. Follow-up time was calculated beginning six months after lymphoma diagnosis until administrative censoring, death, or loss to follow-up. Follow-up was administratively censored on December 31, 2011. Loss to follow-up was assigned based on last date of any clinical activity in CNICS. Mortality rates were calculated as the number of deaths per 100 person-years of follow-up. Kaplan-Meier cumulative mortality curves were used to estimate probability of death beginning six months after lymphoma diagnosis. Differences in cumulative mortality across viremia-6-months categories were evaluated using the log-rank test. Cox proportional hazards modeling was used to estimate the relationship between viremia copy-6-months and mortality, adjusted for sex, race/ethnicity, age, lymphoma diagnosis year, hepatitis B or C co-infection, prior AIDS-defining illness, ART status, CD4 count, and histology. All analyses were conducted using SAS version 9.2. A two-sided alpha value of 0.05 was used to assess statistical significance. Patients were excluded from analyses which included variables for which data were missing.

\section{Results}

Of 24,203 HIV-infected individuals enrolled in CNICS, 482 (2\%) individuals were diagnosed with lymphoma between 1996 and 2011. Of these, 224 (46\%) patients met all criteria for study inclusion. Among excluded patients were 192 (40\%) patients lacking at least two distinct HIV RNA measurements from one month before until seven months after diagnosis, as well as $66(14 \%)$ patients who were not at risk for mortality six months after diagnosis when individuals began to contribute follow-up time. Of these 66 patients, 44 died before six months, 9 were censored before six months, and 13 were not officially enrolled in CNICS until more than six months after lymphoma diagnosis.

The final analytic population had a median age of 43 years [interquartile range (IQR) 3849], $92 \%$ were male, $52 \%$ were white, and $79 \%$ had prior AIDS-defining illnesses. Histologic distribution was 41 (18\%) HL, 106 (47\%) diffuse large B-cell lymphoma (DLBCL), 27 (12\%) Burkitt lymphoma (BL), 18 (8\%) primary CNS lymphoma (PCNSL), and $32(14 \%)$ other NHL. At lymphoma diagnosis, median CD4 count was 148 cells/ mL (IQR 54-322), median nadir CD4 count was 73 cells/mL (IQR 28-177), median HIV RNA was $3.99 \log _{10}$ copies/mL (IQR 2.26-5.16), and the proportion of patients with suppressed HIV RNA was 33\%. CD4 count and HIV RNA measurements at lymphoma diagnosis differed from the diagnosis date by a median of 12 days (IQR 4-25) and 12 days (IQR 4-26) respectively. During the exposure window of interest, patients contributed a median of three HIV RNA measures (IQR 2-4), for a total of 667 measures for the entire study population. The median viremia copy-6-months value for the entire study population was 2.68 
$\log _{10}$ copies x 6-months/mL (IQR 1.85-3.94). There were 64 (29\%) patients who had suppressed HIV RNA throughout the six-month exposure window of interest.

At lymphoma diagnosis, 105 (47\%) patients were on ART for a median duration of 14.7 months (IQR 5.1-36.6). Three months after lymphoma diagnosis $129(58 \%)$ were on ART [48 of 119 (37\%) of those off ARTat lymphoma diagnosis; 81 of 105 (63\%) of those on ARTat lymphoma diagnosis]. Six months after lymphoma diagnosis $158(71 \%)$ were on ART [73 of $119(61 \%)$ of those off ART and 85 of $105(81 \%)$ of those on ART at lymphoma diagnosis]. For patients on ART at lymphoma diagnosis, ART was protease inhibitor (PI)-based for $49(47 \%)$ patients, non-nucleoside reverse transcriptase inhibitor (NNRTI)-based for 40 (38\%), integrase inhibitor (II)-based for 4 (4\%), and another regimen for $12(11 \%)$. For patients off ART, the first ART initiated after lymphoma diagnosis was PI-based for 57 (48\%) patients, NNRTI-based for 49 (41\%), II-based for $3(3 \%)$, and another regimen for $6(5 \%)$. Four patients (3\%) off ART had no subsequent ART initiation recorded.

Baseline characteristics for the entire study population stratified by ART status at lymphoma diagnosis are shown in Table 1. Statistically significant differences between the two groups were noted with respect to histology, lymphoma stage, prior AIDS-defining illness, CD4 count, and HIV RNA. Median HIV viremia copy-6-months among those on ART was 2.22 $\log _{10}$ copies x 6-months/mL (IQR 1.54-3.50) versus 2.97 (IQR 2.33-4.14) for those off ART ( $<0.0001)$. Median HIV RNA values over time, for patients on and off ART, are shown in Fig. 1 with interquartile ranges.

Among the 224 patients, 82 deaths occurred during 851 person-years of follow-up, yielding a mortality rate of 9.6 deaths per 100 person-years [95\% confidence interval (CI) 7.8-12.0]. Cumulative mortality for the entire study population was significantly different when stratified by viremia copy-6-months below or above the median value $\left(2.68 \log _{10}\right.$ copies x 6months $/ \mathrm{mL}$ ), as shown in Fig. 2. Overall survival from six months to five years after lymphoma diagnosis was $64 \%$ (95\% CI 57-71\%). Overall survival from six months to five years after lymphoma diagnosis was 54\% (95\% CI 42-65\%) and 70\% (95\% CI 61-79\%) among patients with viremia copy-6-months values above and below the median, respectively $(\mathrm{p}=0.014)$.

Results of Cox proportional hazards modeling are shown in Table 2. The adjusted hazard ratio (AHR) for mortality for HIV viremia copy-6-months was 1.35 (95\% CI 1.11-1.65), suggesting that exposure to each additional 1 -unit $\log _{10}$ increase in HIV RNA throughout the six months after lymphoma diagnosis, was associated with a 35\% increased risk of subsequent death among six-month survivors. Other risk factors for mortality included older age (AHR 1.37 per decade increase, 95\% CI 1.03-1.83), lymphoma occurrence on ART (AHR 1.63, 95\% CI 1.02-2.63), and lower CD4 count at lymphoma diagnosis (AHR 0.75 per $100 \mathrm{cell} / \mu \mathrm{LL}$ increase, $95 \%$ CI $0.64-0.89$ ).

When HIV RNA at lymphoma diagnosis was included in place of viremia copy-6-months, each $\log _{10}$ increase was associated with an AHR for mortality of 1.11 (95\% CI 0.91-1.34), with other model estimates being similar. When HIV RNA six months after lymphoma diagnosis was included in place of viremia copy-6-months, each $\log _{10}$ increase was associated with an AHR for mortality of 1.32 (95\% CI 1.12-1.56), with other model estimates being similar. When dichotomous HIV RNA $\geq 400 \mathrm{copies} / \mathrm{mL}$ (i.e. unsuppressed versus suppressed) at lymphoma diagnosis was included in place of viremia copy-6-months, being unsuppressed was associated with an AHR for mortality of 1.45 (95\% CI 0.78-2.67), with other model estimates being similar. Likewise, when HIV RNA suppression six months after lymphoma diagnosis was included in place of viremia copy-6-months, being 
unsuppressed was associated with an AHR for mortality of 1.64 (95\% CI 1.03-2.63), with other model estimates being similar. HIV RNA at lymphoma diagnosis and six months after diagnosis were not analyzed together with viremia copy-6-months due to collinearity given the short exposure window during which cumulative HIV viremia was defined. Model estimates were additionally similar when nadir CD4 count was included in place of CD4 count at lymphoma diagnosis, and when cumulative viremia was assessed over nine rather than six months after lymphoma diagnosis.

Lymphoma stage was recorded for 90 (40\%) of patients overall, with $29(32 \%)$ having stage I/II disease and 62 (69\%) having stage III/IV disease. In Cox proportional hazards modeling in the subset of patients with known lymphoma stage, including all covariates as in Table 2 with the addition of stage (I/II versus III/IV), the AHR for mortality for HIV viremia copy-6-months was 1.45 (95\% CI 0.97-2.16), consistent with results in the entire study population and with other model estimates being similar.

AHR estimates for the association between viremia copy-6-months and mortality, adjusted for all covariates as in Table 2, are shown for the full analytic population as well as predefined subgroups in Fig. 3. These analyses show consistent associations between early cumulative HIV viremia and increased mortality, among patients on and off ART, across CD4 strata, and among patients with NHL and HL.

\section{Discussion}

Our study is the first to examine effects of early HIV viremia on survival after HIVassociated lymphoma. The results, drawn from a large multicenter HIV-infected cohort in the United States, suggest that increased viremia during the six months after lymphoma diagnosis is associated with an increased risk of death between six months and five years after diagnosis. For each $\log _{10}$ increase in HIV RNA sustained over the six months immediately after lymphoma diagnosis, we observed a $35 \%$ increase in mortality by five years after lymphoma diagnosis. The significant association of mortality with viremia copy-6-months, as well as cross-sectional HIV RNA six months after lymphoma diagnosis, but not HIV RNA at lymphoma diagnosis, suggest that effective ART during chemotherapy may improve overall survival. Although differences in early cumulative HIV viremia and HIV RNA six months after lymphoma diagnosis may be influenced by patient and disease characteristics other than ART, early and effective ART is likely to be the major factor influencing HIV viremia after lymphoma diagnosis. The association between viremia copy-6-months and increased mortality was consistent between patients on and off ART at lymphoma diagnosis, those with NHL and HL, and across CD4 strata. Other variables associated with mortality were older age, lymphoma occurrence on ART, and lower CD4 count. Our results are consistent with other preliminary data suggesting that concurrent ART is associated with increased rates of complete remission and possibly overall survival among patients with HIV-associated NHL [27].

In the absence of clear evidence, strategies for coadministering ART and chemotherapy have varied, from concurrent ART during chemotherapy to sequential ART after chemotherapy [5-8], with current guidelines making no strong recommendation for either approach [22]. Discontinuing or deferring ART until chemotherapy completion has resulted in long-term survival of $60-70 \%$ for patients with HIV-associated NHL, when treated with the infusional R-EPOCH regimen (rituximab, etoposide, predisone, vincristine, cyclophosphamide, doxorubicin) $[7,8,10]$, which may be superior to R-CHOP (rituximab, cyclophosphamide, doxorubicin, vincristine, prednisone) for patients with HIV [27,28]. Moreover, short-course $\mathrm{R}$-EPOCH developed at NCI is guided by interim response assessment, with an average seven-week delay from chemotherapy initiation until resumption or initiation of ART, and 
only three chemotherapy cycles being administered in up to $70-80 \%$ of patients demonstrated to have a complete response by fluorodeoxyglucose positron emission tomography (FDG-PET) after two cycles $[8,10]$. However, results from this strategy have been published for only 33 clinical trial participants treated at a single center, and without a comparison group receiving identical chemotherapy with concurrent ART [8]. Additionally, recent AMC studies have typically administered six cycles even when using R-EPOCH and DR-COP (pegylated liposomal doxorubicin, rituximab, cyclophosphamide, vincristine, prednisone) [6,9]. If strictly sequential ART after chemotherapy is recommended, this more standard approach will result in longer periods off ART.

Patients with HIV-associated lymphoma continue to present with significant immunosuppression in the ART era [5,7-9,20,21]. For most OIs, deferring ART by even 410 weeks results in an increased risk of AIDS or death, suggesting that delaying ART for weeks or months until chemotherapy is complete may not be inconsequential, particularly for severely immunosuppressed patients [14-17]. For patients receiving ART, episodic CD4 count-guided ART interruption has been shown in a randomized clinical trial to increase risk of OI or death compared with continuous ART [29]. Although long-term effects of a single fixed-duration ART interruption during chemotherapy remain unknown, short-term effects of ART interruption include marked CD4 count decreases and HIV RNA increases [30-33]. Finally, in an analogous condition PTLD, consensus guidelines recommend reduction of immunosuppression as initial therapy, which can lead to complete remissions even without chemoimmunotherapy in some patients [22], again suggesting that early ART-mediated immune reconstitution among HIV-infected lymphoma patients may be beneficial.

Concerns previously raised regarding interactions of ART with chemotherapy are based on studies of older ART regimens [10-13], whereas current regimens typically include lower ritonavir doses, newer agents such as integrase inhibitors, and agents for which pharmacokinetic profiles are better defined. Studies proposing specific anti-apoptotic effects of protease inhibitors likely reflect intracellular drug concentrations not achieved during actual patient use [12,13], and contradict empirical demonstrations of protease inhibitor antiproliferative effects in vitro against melanoma, lung cancer, and breast cancer cell lines, by inhibition of signaling pathways involving phosphatidylinositol 3-kinase (PI3K), AKT, and heat shock protein 90 (HSP90) [34-36].

The observation of increased mortality among patients developing lymphoma on ART has been previously reported [21]. Lymphoma which develops on ART may be biologically distinct from lymphoma occurring off ART, analogous to early and late PTLD, which differ in EBV association, clinical behavior, and gene expression patterns [37-40]. Such differences in tumor biology have not been investigated for HIV-associated lymphoma. Other possible explanations include patients already on ARTat lymphoma diagnosis not benefiting from positive effects on survival conferred by ART initiation, in addition to lymphoma treatment. Exposure to ART at lymphoma diagnosis is also a marker of longer duration of HIV infection and more advanced HIV illness, which may not be adequately adjusted for by measures of HIV disease severity. Additionally, social and behavioral differences impacting survival, including adherence, may exist between patients developing lymphoma on and off ART.

Our research has several limitations. First, data are observational and estimates regarding early HIV viremia effects on mortality may be subject to unmeasured confounding. Second, detailed information regarding lymphoma treatment, stage, and other prognostic features were not available for most patients. Third, cause of death was unknown, and our analyses focused on overall survival, although patients with HIV-associated lymphoma are at risk for competing causes of death. Finally, although drawn from one of the largest observational 
cohorts of patients with HIV-associated lymphoma, our analyses were restricted to a smaller subset of patients in whom early cumulative HIV viremia after lymphoma diagnosis could be reliably assessed, and who remained at risk for mortality six months after lymphoma diagnosis.

Despite these limitations, our study has several strengths. To our knowledge, this is the first study to examine the association between early HIV viremia and subsequent mortality after lymphoma diagnosis. Patients studied represent a large and diverse HIV-infected population in routine care across the United States. Mortality assessment used active and passive surveillance, leading to near-complete ascertainment.

In conclusion, higher HIV viremia during the six months after lymphoma diagnosis was associated with an increased risk of subsequent death in our analyses of United States HIVinfected lymphoma patients surviving at least six months. These findings support treatment strategies incorporating early ART with maximal HIV suppression, concurrently with chemotherapy, for patients with HIV-associated lymphoma.

\section{Acknowledgments}

S.G. worked closely with S.N., C.J.A., K.L.R., and J.J.E. to design the study. S.G., S.N., and M.R.P. acquired the data from the CNICS data management core. M.R.P. compiled the study database. S.G., M.R.P., E.L.Y., S.R.C., and S.N. collaborated on statistical analyses. S.G. and M.R.P. participated in writing the manuscript. All authors reviewed and commented on the manuscript, and approved its final submission.

These findings are presented on behalf of CNICS, an NIH-funded program (R24 AI067039) made possible by the National Institute of Allergy and Infectious Diseases. This research was also supported by the University of North Carolina CFAR, an NIH-funded program (P30 AI50410), the University of Alabama at Birmingham CFAR (P30AI027767), and the Case Western Reserve University CFAR (P30 AI36219). Additional support was provided by a UNC Lineberger Comprehensive Cancer Center Developmental Research Award. Dr. Gopal is additionally supported by the Fogarty International Center of the NIH through the Fogarty Global Health Fellows Program (1R25TW009340-01) and an International Scientist Development Award (1K01TW009488-01), as well as an AIDS Malignancy Consortium Fellowship Award.

The authors would like to thank all CNICS investigators and data management teams from the eight sites who contributed to the completion of this study at Case Western Reserve University, University of Alabama at Birmingham, University of California, San Francisco, University of Washington, University of California, San Diego, Fenway Health, University of North Carolina, and Johns Hopkins University. The authors additionally wish to acknowledge Donna Porter for her significant contributions.

\section{References}

1. Simard EP, Engels EA. Cancer as a cause of death among people with AIDS in the United States. Clin Infect Dis. 2010; 51:957-962. [PubMed: 20825305]

2. Bonnet F, Burty C, Lewden C, Costagliola D, May T, Bouteloup V, et al. Changes in cancer mortality among HIV-infected patients: the Mortalite 2005 Survey. Clin Infect Dis. 2009; 48:633639. [PubMed: 19202627]

3. Causes of death in HIV-1-infected patients treated with anti-retroviral therapy, 1996-2006: collaborative analysis of 13 HIV cohort studies. Clin Infect Dis. 2010; 50:1387-1396. [PubMed: 20380565]

4. Achenbach CJ, Cole SR, Kitahata MM, Casper C, Willig JH, Mugavero MJ, et al. Mortality after cancer diagnosis in HIV-infected individuals treated with antiretroviral therapy. AIDS. 2011; 25:691-700. [PubMed: 21160411]

5. Kaplan LD, Lee JY, Ambinder RF, Sparano JA, Cesarman E, Chadburn A, et al. Rituximab does not improve clinical outcome in a randomized phase 3 trial of CHOP with or without rituximab in patients with HIV-associated non-Hodgkin lym-phoma: AIDS-Malignancies Consortium Trial 010. Blood. 2005; 106:1538-1543. [PubMed: 15914552] 
6. Levine AM, Noy A, Lee JY, Tam W, Ramos JC, Henry DH, et al. Pegylated liposomal doxorubicin, rituximab, cyclophosphamide, vincristine, and prednisone in AIDS-related lymphoma: AIDS Malignancy Consortium Study 047. J Clin Oncol. 2013; 31:58-64. [PubMed: 23169503]

7. Little RF, Pittaluga S, Grant N, Steinberg SM, Kavlick MF, Mitsuya H, et al. Highly effective treatment of acquired immunodeficiency syndrome-related lymphoma with dose-adjusted EPOCH: impact of antiretroviral therapy suspension and tumor biology. Blood. 2003; 101:4653-4659. [PubMed: 12609827]

8. Dunleavy K, Little RF, Pittaluga S, Grant N, Wayne AS, Carras-quillo JA, et al. The role of tumor histogenesis, FDG-PET, and short-course EPOCH with dose-dense rituximab (SC-EPOCH-RR) in HIV-associated diffuse large B-cell lymphoma. Blood. 2010; 115:3017-3024. [PubMed: 20130244]

9. Sparano JA, Lee JY, Kaplan LD, Levine AM, Ramos JC, Ambinder RF, et al. Rituximab plus concurrent infusional EPOCH chemotherapy is highly effective in HIV-associated B-cell nonHodgkin lymphoma. Blood. 2010; 115:3008-3016. [PubMed: 20023215]

10. Dunleavy K, Wilson WH. How I treat HIV-associated lymphoma. Blood. 2012; 119:3245-3255. [PubMed: 22337719]

11. Ratner L, Lee J, Tang S, Redden D, Hamzeh F, Herndier B, et al. Chemotherapy for human immunodeficiency virus-associated non-Hodgkin's lymphoma in combination with highly active antiretroviral therapy. J Clin Oncol. 2001; 19:2171-2178. [PubMed: 11304769]

12. Phenix BN, Lum JJ, Nie Z, Sanchez-Dardon J, Badley AD. Antiapoptotic mechanism of HIV protease inhibitors: preventing mitochondrial transmembrane potential loss. Blood. 2001; 98:1078-1085. [PubMed: 11493454]

13. Phenix BN, Cooper C, Owen C, Badley AD. Modulation of apoptosis by HIV protease inhibitors. Apoptosis. 2002; 7:295-312. [PubMed: 12101389]

14. Zolopa A, Andersen J, Powderly W, Sanchez A, Sanne I, Suckow C, et al. Early antiretroviral therapy reduces AIDS progression/ death in individuals with acute opportunistic infections: a multicenter randomized strategy trial. PLoS One. 2009; 4:e5575. [PubMed: 19440326]

15. Blanc FX, Sok T, Laureillard D, Borand L, Rekacewicz C, Nerrienet E, et al. Earlier versus later start of antiretroviral therapy in HIV-infected adults with tuberculosis. N Engl J Med. 2011; 365:1471-1481. [PubMed: 22010913]

16. Abdool Karim SS, Naidoo K, Grobler A, Padayatchi N, Baxter C, Gray AL, et al. Integration of antiretroviral therapy with tuberculosis treatment. N Engl J Med. 2011; 365:1492-1501. [PubMed: 22010915]

17. Havlir DV, Kendall MA, Ive P, Kumwenda J, Swindells S, Qasba SS, et al. Timing of antiretroviral therapy for HIV-1 infection and tuberculosis. N Engl J Med. 2011; 365:1482-1491. [PubMed: 22010914]

18. Torok ME, Yen NT, Chau TT, Mai NT, Phu NH, Mai PP, et al. Timing of initiation of antiretroviral therapy in human immunodeficiency virus (HIV)-associated tuberculous meningitis. Clin Infect Dis. 2011; 52:1374-1383. [PubMed: 21596680]

19. National Institute for Allergy and Infectious Diseases. [Accessed 2013 January 23] HIV Treatment Study in Patients with Cryptococcal Meningitis Ends Enrollment Early, Higher Mortality Rate Found with Early Antiretroviral Therapy. http://www.niaid.nih.gov/news/newsre-leases/2012/ Pages/COAT.aspx

20. Chao C, Xu L, Abrams D, Leyden W, Horberg M, Towner W, et al. Survival of non-Hodgkin lymphoma patients with and without HIV infection in the era of combined antiretroviral therapy. AIDS. 2010; 24:1765-1770. [PubMed: 20453630]

21. Gopal S, Patel M, Yanik EL, Cole SR, Achenbach CJ, Napravnik S, et al. Temporal trends in presentation and survival for HIV-associated lymphoma in the antiretroviral therapy era. J Natl Cancer Inst. 2013 [In press].

22. National Comprehensive Cancer Network. [Accessed 2012 December 5] NCCN Clinical Practice Guidelines in Oncology: Non-Hodgkin's Lymphomas Version 2.2012. http://www.nccn.org/ professionals/physician_gls/pdf/nhl.pdf

23. Kitahata MM, Rodriguez B, Haubrich R, Boswell S, Mathews WC, Lederman MM, et al. Cohort profile: the Centers for AIDS Research Network of Integrated Clinical Systems. Int J Epidemiol. 2008; 37:948-955. [PubMed: 18263650] 
24. Achenbach CJ, Cole SR, Kitahata MM, Casper C, Willig JH, Mugavero MJ, Saag MS. Mortality after cancer diagnosis in HIV-infected individuals treated with antiretroviral therapy. AIDS. 2011; 25:691-700. [PubMed: 21160411]

25. Cole SR, Napravnik S, Mugavero MJ, Lau B, Eron JJ Jr, Saag MS. Copy-years viremia as a measure of cumulative human immunodeficiency virus viral burden. Am J Epidemiol. 2010; 171:198-205. [PubMed: 20007202]

26. Mugavero MJ, Napravnik S, Cole SR, Eron JJ, Lau B, Crane HM, et al. Viremia copy-years predicts mortality among treatmentnaive HIV-infected patients initiating antiretroviral therapy. Clin Infect Dis. 2011; 53:927-935. [PubMed: 21890751]

27. Barta, SK.; Xue, X.; Wang, D.; Tamari, R.; Lee, JY.; Mounier, N., et al. A Pooled Analysis of 1,546 Patients with HIV-Associated Lym-phoma: Assessment of Lymphoma-, HIV-, and Treatment-Specific Factors On Clinical Outcomes [Abstract]; Atlanta, Geogia. Presented at Annual Meeting of the American Society of Hematology; Dec 10. 2012 Abstract 3682

28. Barta SK, Lee JY, Kaplan LD, Noy A, Sparano JA. Pooled analysis of AIDS malignancy consortium trials evaluating rituximab plus CHOP or infusional EPOCH chemotherapy in HIVassociated non-Hodgkin lymphoma. Cancer. 2011; 118:3977-3983. [PubMed: 22180164]

29. El-Sadr WM, Lundgren JD, Neaton JD, Gordin F, Abrams D, Arduino RC, et al. CD4+ countguided interruption of antiretroviral treatment. N Engl J Med. 2006; 355:2283-2296. [PubMed: 17135583]

30. Achenbach CJ, Till M, Palella FJ, Knoll MD, Terp SM, Kalnins AU, et al. Extended antiretroviral treatment interruption in HIV-infected patients with long-term suppression of plasma HIV RNA. HIV Med. 2005; 6:7-12. [PubMed: 15670246]

31. Ruiz L, Carcelain G, Martinez-Picado J, Frost S, Marfil S, Paredes R, et al. HIV dynamics and Tcell immunity after three structured treatment interruptions in chronic HIV-1 infection. AIDS. 2001; 15:F19-F27. [PubMed: 11416734]

32. Garcia F, Plana M, Ortiz GM, Bonhoeffer S, Soriano A, Vidal C, et al. The virological and immunological consequences of structured treatment interruptions in chronic HIV-1 infection. AIDS. 2001; 15:F29-F40. [PubMed: 11416735]

33. Hatano H, Vogel S, Yoder C, Metcalf JA, Dewar R, Davey RT Jr, et al. Pre-HAART HIV burden approximates post-HAART viral levels following interruption of therapy in patients with sustained viral suppression. AIDS. 2000; 14:1357-1363. [PubMed: 10930150]

34. Shim JS, Rao R, Beebe K, Neckers L, Han I, Nahta R, et al. Selective Inhibition of HER2-Positive Breast Cancer Cells by the HIV Protease Inhibitor Nelfinavir. J Natl Cancer Inst. 2012; 104:15761590. [PubMed: 23042933]

35. Yang Y, Ikezoe T, Nishioka C, Bandobashi K, Takeuchi T, Adachi Y, et al. NFV, an HIV-1 protease inhibitor, induces growth arrest, reduced Akt signalling, apoptosis and docetaxel sensitisation in NSCLC cell lines. Br J Cancer. 2006; 95:1653-1662. [PubMed: 17133272]

36. Jiang W, Mikochik PJ, Ra JH, Lei H, Flaherty KT, Winkler JD, et al. HIV protease inhibitor nelfinavir inhibits growth of human melanoma cells by induction of cell cycle arrest. Cancer Res. 2007; 67:1221-1227. [PubMed: 17283158]

37. Knight JS, Tsodikov A, Cibrik DM, Ross CW, Kaminski MS, Blayney DW. Lymphoma after solid organ transplantation: risk, response to therapy, and survival at a transplantation center. J Clin Oncol. 2009; 27:3354-3362. [PubMed: 19451438]

38. Leblond V, Davi F, Charlotte F, Dorent R, Bitker MO, Sutton L, et al. Posttransplant lymphoproliferative disorders not associated with Epstein-Barr virus: a distinct entity? J Clin Oncol. 1998; 16:2052-2059. [PubMed: 9626203]

39. Nelson BP, Nalesnik MA, Bahler DW, Locker J, Fung JJ, Swer-dlow SH. Epstein-Barr virusnegative post-transplant lymphoproliferative disorders: a distinct entity? Am J Surg Pathol. 2000; 24:375-385. [PubMed: 10716151]

40. Craig FE, Johnson LR, Harvey SA, Nalesnik MA, Luo JH, Bhat-tacharya SD, et al. Gene expression profiling of Epstein-Barr virus-positive and -negative monomorphic B-cell posttransplant lymphoproliferative disorders. Diagn Mol Pathol. 2007; 16:158-168. [PubMed: 17721324] 


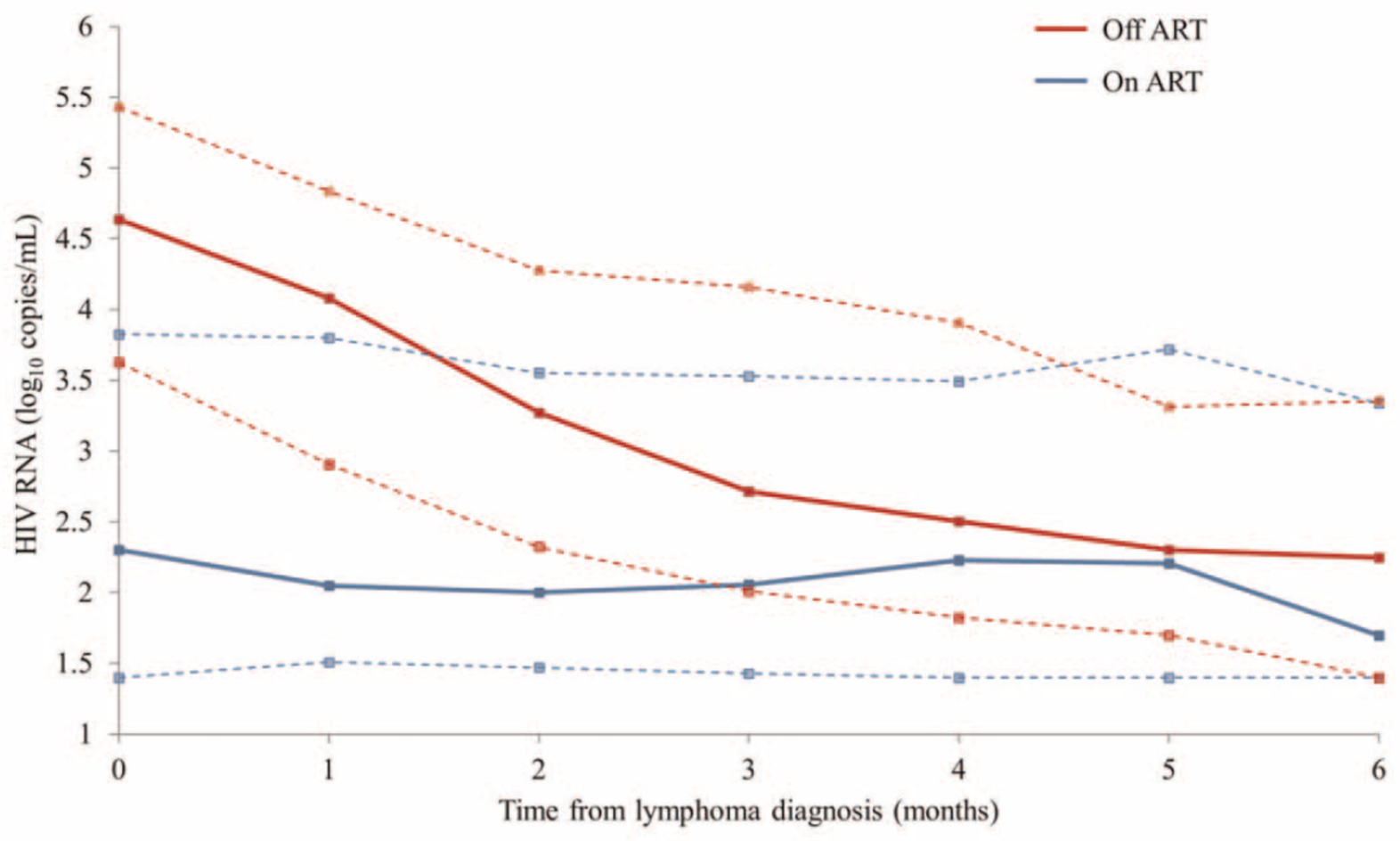

Fig. 1.

Median HIV RNA and interquartile range for $224 \mathrm{HIV}$-infected adults in CNICS during the 6 months after lymphoma diagnosis, stratified by antiretroviral therapy (ART) status at lymphoma diagnosis. 


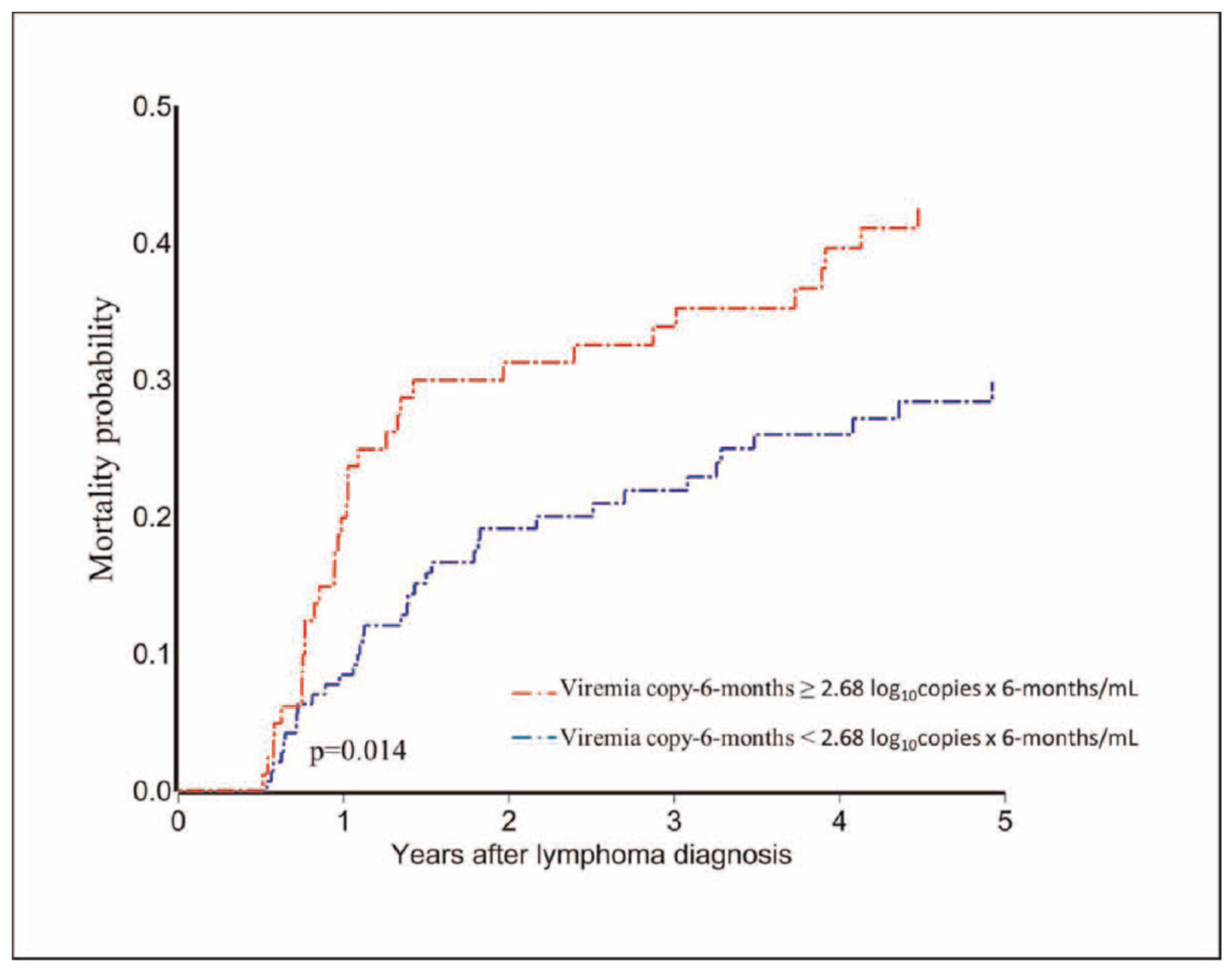

Fig. 2.

Cumulative mortality after lymphoma diagnosis for $224 \mathrm{HIV}$-infected adults in CNICS, stratified by HIV viremia copy- 6 months $\left(\log _{10}\right.$ copies $\times 6$-months $\left./ \mathrm{mL}\right)$. 


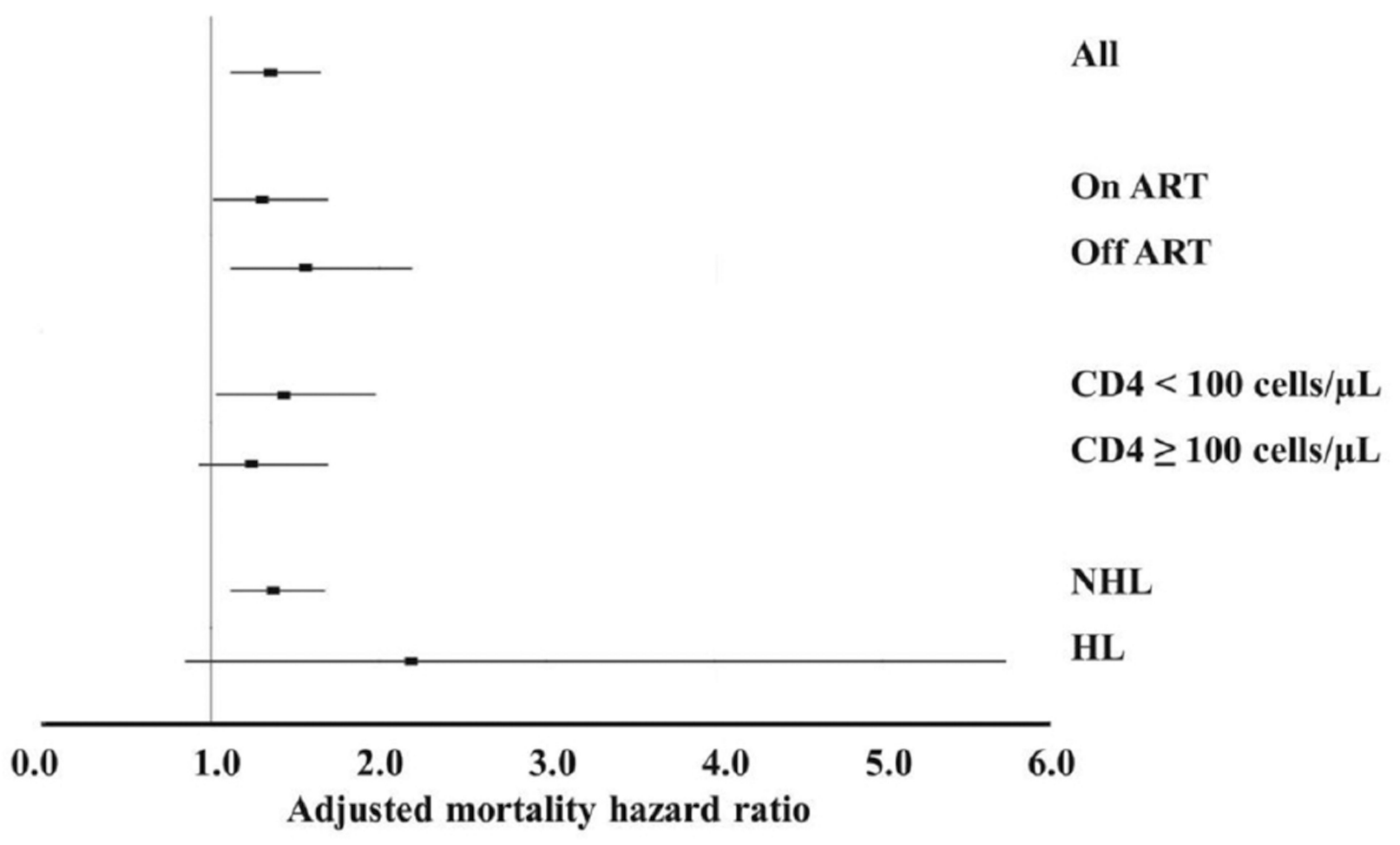

Fig. 3.

Adjusted mortality hazard ratios for HIV viremia-copy-6 months among HIV-infected adults in CNICS with lymphoma between 1996 and 2011, stratified by patient characteristics at lymphoma diagnosis. ART, antiretroviral therapy; HL, Hodgkin lymphoma; NHL, nonHodgkin lymphoma. 
Table 1

Characteristics of 224 HIV-infected adults in CNICS with lymphoma between 1996 and 2011, stratified by ART status at lymphoma diagnosis.

\begin{tabular}{|c|c|c|c|}
\hline & On ART & Off ART & p value \\
\hline Total, n (\%) & $105(46.9)$ & $119(53.1)$ & - \\
\hline Age (years) at lymphoma diagnosis, mean (SD) & $44.2(7.7)$ & $42.4(9.2)$ & 0.12 \\
\hline Male, n (\%) & $99(94.3)$ & 109 (91.6) & 0.44 \\
\hline \multicolumn{4}{|l|}{ Race/ethnicity ${ }^{a}$} \\
\hline White, $\mathrm{n}(\%)$ & $59(56.2)$ & $59(50.4)$ & 0.39 \\
\hline Black, n (\%) & $28(26.7)$ & $32(27.4)$ & 0.91 \\
\hline Other, $\mathrm{n}(\%)$ & $18(17.1)$ & $26(22.2)$ & 0.34 \\
\hline Lymphoma diagnosis year, median (IQR) & $2004(2001-2007)$ & $2004(2002-2007)$ & 0.82 \\
\hline \multicolumn{4}{|l|}{ Lymphoma category } \\
\hline $\mathrm{HL}, \mathrm{n}(\%)$ & $30(28.6)$ & $11(9.2)$ & 0.0002 \\
\hline DLBCL, n (\%) & $45(42.9)$ & $61(51.3)$ & 0.21 \\
\hline $\mathrm{BL}, \mathrm{n}(\%)$ & $8(7.6)$ & $19(16.0)$ & 0.056 \\
\hline PCNSL, n (\%) & $4(3.8)$ & $14(11.8)$ & 0.029 \\
\hline Other NHL, n (\%) & $18(17.1)$ & $14(11.8)$ & 0.25 \\
\hline Lymphoma stage I/II, n (\%) ${ }^{a}$ & $19(47.5)$ & $10(19.6)$ & 0.0046 \\
\hline Hepatitis B/C co-infection & $22(21.0)$ & $30(25.2)$ & 0.45 \\
\hline AIDS illness prior to lymphoma diagnosis, $\mathrm{n}(\%)$ & $90(85.7)$ & $87(73.1)$ & 0.021 \\
\hline $\mathrm{CD} 4$ count $($ cells $/ \mu \mathrm{L})$ at lymphoma diagnosis, median $(\mathrm{IQR})^{a}$ & $188(78-339)$ & $123(49-287)$ & 0.051 \\
\hline $\mathrm{CD} 4$ percentage at lymphoma diagnosis, median $(\mathrm{IQR})^{a}$ & $18.0(10.4-26.9)$ & $11.3(6.0-20.0)$ & 0.0007 \\
\hline $\mathrm{CD} 4$ count $(\mathrm{cells} / \mathrm{\mu L})$ nadir, median $(\mathrm{IQR})^{a}$ & $74(27-198)$ & $72(29-153)$ & 0.76 \\
\hline HIV RNA $\left(\log _{10}\right.$ copies $\left./ \mathrm{mL}\right)$ at lymphoma diagnosis, median $(\mathrm{IQR})^{a}$ & $2.30(1.40-3.81)$ & $4.83(3.98-5.57)$ & $<0.0001$ \\
\hline HIV RNA $<400$ copies/mL at lymphoma diagnosis, $\mathrm{n}(\%)^{a}$ & $64(63.4)$ & $7(6.1)$ & $<0.0001$ \\
\hline HIV viremia copy-6-months $\left(\log _{10}\right.$ copies x 6-months/mL) & $2.22(1.54-3.50)$ & $2.97(2.33-4.14)$ & $<0.0001$ \\
\hline
\end{tabular}

ART, antiretroviral therapy; BL, Burkitt lymphoma; DLBCL, diffuse large B-cell lymphoma; HL, Hodgkin lymphoma; PCNSL, primary CNS lymphoma.

${ }^{a} \mathrm{~N}(\%)$ missing observations on ART/off ART: Race/ethnicity=0(0\%)/2(1.7\%); Lymphoma stage $1 / 465(61.9 \%) / 68(57.1 \%)$; CD4 count at lymphoma diagnosis $=4(3.8 \%) / 2(1.7 \%)$; CD4 percentage at lymphoma diagnosis $=8(7.6 \%) / 9(7.6 \%)$; $\mathrm{CD} 4$ count nadir $=2(1.9 \%) / 2(1.7 \%)$; HIV RNA at lymphoma diagnosis $=4(3.8 \%) / 5(4.2 \%)$. 
Table 2

Adjusted mortality hazard ratios for 216 HIV-infected adults in the CNICS cohort with lymphoma between 1996 and 2011.a

\begin{tabular}{|c|c|c|c|}
\hline & Adjusted hazard ratio & $95 \% \mathrm{CI}$ & p value \\
\hline Male sex & 1.72 & $0.56-5.25$ & 0.34 \\
\hline Non-white ethnicity & 0.65 & $0.40-1.04$ & 0.074 \\
\hline Age at lymphoma diagnosis, per decade & 1.37 & $1.03-1.83$ & 0.033 \\
\hline \multicolumn{4}{|l|}{ Lymphoma diagnosis year } \\
\hline $1996-2000$ & Reference & - & - \\
\hline $2001-2005$ & 1.00 & $0.56-1.77$ & 0.99 \\
\hline 2006-2009 & 1.13 & $0.56-2.29$ & 0.74 \\
\hline Hepatitis B/C co-infection & 1.25 & $0.71-2.18$ & 0.44 \\
\hline AIDS illness prior to lymphoma diagnosis & 1.16 & $0.66-2.07$ & 0.61 \\
\hline ART at lymphoma diagnosis & 1.63 & $1.02-2.63$ & 0.043 \\
\hline CD4 count at lymphoma diagnosis, per 100 cells $/ \mu \mathrm{L}$ & 0.75 & $0.64-0.89$ & 0.0008 \\
\hline \multicolumn{4}{|l|}{ Lymphoma category } \\
\hline PCNSL (reference) & - & - & - \\
\hline $\mathrm{HL}$ & 0.45 & $0.14-1.44$ & 0.18 \\
\hline DLBCL & 0.94 & $0.38-2.32$ & 0.89 \\
\hline BL & 0.93 & $0.26-3.31$ & 0.92 \\
\hline Other NHL & 0.85 & $0.31-2.34$ & 0.75 \\
\hline HIV viremia copy-6-months $\left(\log _{10}\right.$ copies $\times 6$-months $/ \mathrm{mL}$ ) & 1.35 & $1.11-1.65$ & 0.0029 \\
\hline
\end{tabular}

ART, antiretroviral therapy; BL, Burkitt lymphoma; DLBCL, diffuse large B-cell lymphoma; HL, Hodgkin lymphoma; NHL, Non-Hodgkin lymphoma; PCNSL, primary CNS lymphoma.

$a_{8}$ of 224 patients (3.6\%) with missing data were excluded from Cox proportional hazards modeling. 